\title{
The weakest link: The decline of the surface transportation network
}

\author{
Feng Xie $^{\mathrm{a}, *}$, David Levinson ${ }^{\mathrm{b}, 1}$

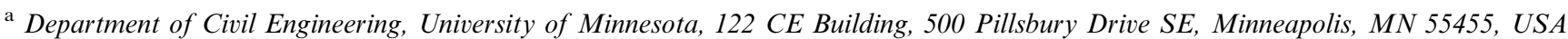 \\ ${ }^{\mathrm{b}}$ Department of Civil Engineering, University of Minnesota, USA
}

Received 26 August 2005; received in revised form 12 July 2006; accepted 15 September 2006

\begin{abstract}
This study explores the economic mechanisms behind the decline of a surface transportation network, based on the assumption that the decline phase is a spontaneous process driven by decentralized decisions of individual travelers and privatized links. A simulation model is developed with a degeneration process by which the weakest link is removed iteratively from the network. Experiments reveal how the economic efficiency of a network evolves during the degeneration process and suggest an "optimal" degenerated network could be derived during the decline phase in terms of maximizing total social welfare.
\end{abstract}

(C) 2006 Elsevier Ltd. All rights reserved.

Keywords: Decline; Transportation network; Simulation; Welfare; Accessibility

\section{Introduction}

Transportation systems, like other economic entities, decline when they become profitless. Examples include the canal system in England (Fullerton, 1975), the interurban in North America (Hilton and Due, 1960), and more recently, the networks of Amtrak and Greyhound in the United States. According to Garrison and Levinson (2006), there were 416,000 km of railroad in the United States by 1920, which has shrunk to about $272,000 \mathrm{~km}$ in the present.

The decline of a transportation system can be caused by a wide variety of technical, economic, social, and political reasons. From an economic perspective, this study focuses on a surface transportation system that fails to recover its costs from its income and thus shrinks. A planar transportation system is usually embodied as a network in which locations and facilities (nodes) are connected by trails, tracks or paved roads (links). If the network cannot earn its keep as it evolves, it becomes economically inefficient and some of the weakest links have to be abandoned.

\footnotetext{
${ }^{*}$ Corresponding author. Tel.: +1 612626 0024; fax: +1 6126267750 .

E-mail addresses: xiex0055@umn.edu (F.Xie), dlevinson@umn.edu (D. Levinson).

${ }^{1}$ Tel.: +1 6126256354 .
} 
The purpose of this study is to investigate the economic mechanisms behind the decline of a transportation network and their consequences. The following section presents a review of related literature. In the model section the simulation model is presented, followed by performance measures from different perspectives. Then simulation experiments are outlined and results presented. The conclusion part summarizes our findings and suggests implications for future research.

\section{Literature review}

Life cycle theory explains the decline of a transportation network at a macroscopic level (Mensch, 1979; Nakicenovic, 1988; Garrison and Levinson, 2006). According to life cycle theory, most technologies (e.g. transportation modes) experience the phases of birth, growth, maturity, and decline in their evolutions. The S-curve technique provides a tool to describe the life cycle of a transportation mode collectively, relating the mode's share of final market and time. However, it is still insufficient to understand the microscopic mechanisms behind the evolution of transportation systems.

The evolution of a transportation network has traditionally been attributed to the top-down decisions made by central agencies, such as engineers, planners, and policy makers. Since the 1970s, planners and transportation researchers have sought to find optimal improvements to a network, in order to achieve stated planning objectives given the constraints of available resources, well known as "Network Design Problems" (Boyce et al., 1974; Yang and Bell, 1998). Based on the assumption that a network can be optimized, Vaughan (1987) discussed the design of optimum network geometry, while Schweitzer et al. (1998) applied two strategies to find optimal decisions for the evolution of a road network.

Recent studies, however, have suggested that the macro characteristics of a complex system, rather than being designed by authorities, could be shaped by simple, perhaps myopic behaviors of underlying entities (Schelling, 1978; Krugman, 1996). The concepts of reinforcement and degeneration have then been introduced to interpret the evolution of complex systems as a bottom-up agent-based process in many areas (Barabasi et al., 1999; Barabasi and Bonabeau, 2003; Newman, 2003), claiming that a complex network evolves through interactions with its environment with successful agents of the system reinforced while less successful ones deprecated or abandoned.

Among the few studies that interpret the evolution of a transportation network as an agent-based process, Helbing et al. (1997) simulates the evolution of human trails in urban green spaces, in which frequently used trails are reinforced and made more attractive while rarely used trails are destroyed by "the weathering effects". Yamins et al. (2003) present a simulation of road dynamics on a land use lattice according to simple growing rules. Yerra and Levinson (2005) and Levinson and Yerra (2006) model each link in a road network as an agent that can invest autonomously so that profitable links get expanded while profitless links get contracted. Zhang and Levinson (2004) examined the growth of a real-world congesting network - the Twin Cities road network. Based on the network topology in 1978, simulation experiments were carried out to "predict" road expansions in 20 years under different initial conditions and constraints. Comparing predicted 1998 networks to the real one, they found like road hierarchies emerged especially for low-level roads, whether they started from the real 1978 network with observed road capacities or a hypothetical network with uniform capacities. All these studies, however, assume a fixed network structure, thus not allowing less developed links to be fully degenerated or abandoned.

Questions arise accordingly when we try to interpret the decline phase of a transportation network as a completely bottom-up process that is driven by decisions made by underlying agents such as travelers and privatized links: how could this process be modeled in a as possible as simple form so that simulation can be executed for sufficient iterations in an acceptable duration, while incorporating all the essential components such as land use, traffic flow, agent behaviors, and network decline? How will a declining network evolve as a decentralized and competitive market? How do economists, policy makers, and transportation planners evaluate market performance differently, and can they at least approach their respective objectives by interfering with the market during the decline phase?

To answer these questions, a simulation model which we refer to as the degeneration model is developed to implement the abandonment of links in a network by introducing a process in which the weakest links are eliminated each round based on a myopic evaluation of individual operating performance. Following Levin- 
son and Yerra (2006), individual travelers choose their destinations and routes to minimize their travel cost while independent link agents invest (disinvest) in themselves according to their revenue and costs associated with predicted traffic on the network.

\section{Model}

Fig. 1 illustrates the flowchart of the degeneration model. Network evolution has been modeled as an iterative dynamic process, which includes five sequential steps for each iteration: exogenous input, degeneration, land use dynamics, travel demand dynamics, and investment. These steps are explained as follows.

The exogenous inputs include a base transportation network structure and a land use layer, specified initial land use data, and initial link speeds. Our study, following Levinson and Yerra (2006), models the land use layer as a grid of land blocks (i.e., land use cells). Each land use cell holds the information of its location, population density and market density. The transportation network is modeled as a directed network overlaying the land use layer. Since the nodes and links are removable during the degeneration process, the collection of existing nodes and that of existing links in a directed network are represented by dynamic sets $\left\{\boldsymbol{V}^{i}\right\}$ and $\left\{\boldsymbol{E}^{i}\right\}$,

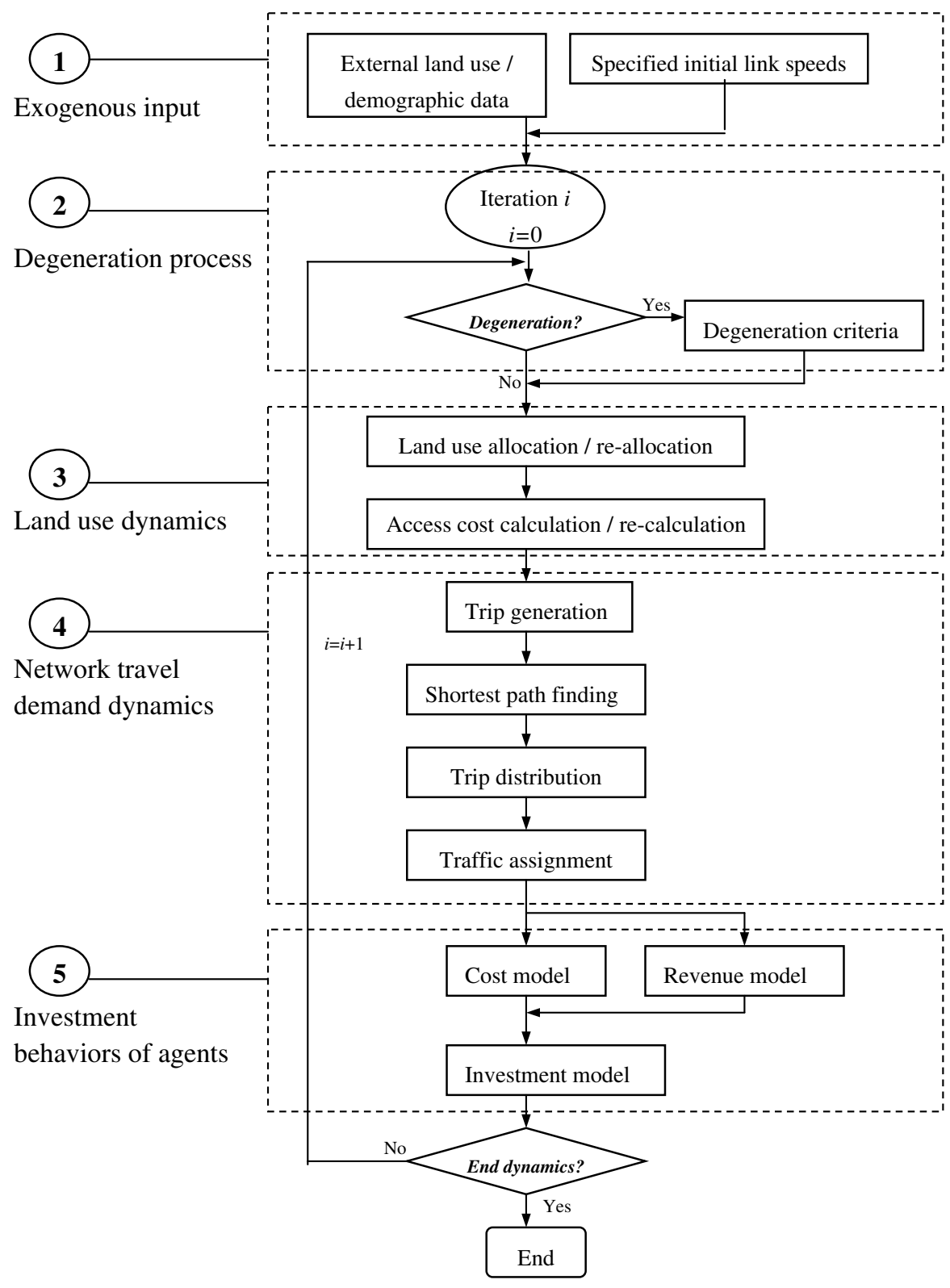

Fig. 1. Flowchart of the transportation network degeneration model. 
respectively, where $i$ is the time period in the simulation. The network layer and land use layer are connected dynamically and are able to affect each other through network nodes. The land use layer, however, is fixed in this study to understand network dynamics in isolation.

The degeneration process is characterized by the degeneration criteria, which specify simple rules to select and kill less developed links. When all links connected to a node are killed, the node may be killed, depending on specific degeneration criteria. The degeneration criteria used in this study will be specified later.

For simplicity this study assumes that trips produced from and attracted to land use cells are subject to a specific distribution and only tests the uniform distribution in this study. Each land use cell is allocated to the closest network node. The time cost of accessing the nearest network node from a land use cell is called the "access cost", which will be explained later. Note that if a network node is eliminated in the degeneration process, the land use cells previously allocated to this node will be re-allocated to other nodes. Consequently, the access costs of network nodes also need to be re-calculated.

This study incorporates a simplified travel demand model which basically follows the classic four-step model (Ortuzar and Willumsen, 2001) with two important simplifications. First, a single mode is considered throughout, and thus modal choice is ignored. Second, congestion effects are ignored in traffic assignment. Although the congestion may occur in the short run given relatively inelastic road capacity, in the long run the model assumes that the congested (heavily traveled) links generate excess revenue (revenue greater than existing maintenance and operation costs) and they can invest their excess income to improve their capacities. Therefore, instead of modeling the congestion effect using the link performance function (i.e., link flow-cost function), we adopt an all-or-nothing assignment and model the economic investment behaviors of links from the long-term perspective of transportation network dynamics. The simplified travel demand model includes four component models: trip generation, shortest path finding, trip distribution, and traffic assignment.

In the trip generation step the total trips produced and attracted from a network node are calculated by summing up trips of all the land use cells allocated to this network node.

Dijkstra's Algorithm (Chachra et al., 1979) is adopted to find the shortest path, i.e., the path with the lowest generalized cost, from an origin node to all other nodes in the network. Let $c_{a}^{i}$ represent the generalized cost on link $a$ for iteration $i$. This cost is calculated as the linear combination of time cost and monetary cost (in this study, toll represents all monetary costs spent on travel) as shown in the following equation:

$$
c_{a}^{i}=\eta l_{a} / v_{a}^{i}+\tau\left(l_{a}\right)^{\rho_{1}}\left(f_{a}^{i}\right)^{\rho_{2}}\left(v_{a}^{i}\right)^{\rho_{3}} \quad \forall a \in\left\{A^{i}\right\},
$$

where $\eta$ is time value, $l_{a}$ is the length of link $a, f_{a}^{i}$, and $v_{a}^{i}$ are the average flow (volume) and average speed of link $a$ for iteration $i$. Coefficients $\tau, \rho_{1}, \rho_{2}$, and $\rho_{3}$ are the toll rate, length coefficient, flow coefficient, and speed coefficient specified in the revenue model, respectively.

The bolded curve in Fig. 2 represents the shortest path originated from node $R$ to all other nodes. The path from $R$ to $S$ can be abstracted as $\left\{\boldsymbol{P}_{R S}\right\}$, i.e., the set of links along the shortest path from origin $R$ to destination $S$. Suppose $m$ land use cells $r_{1}, r_{2}, \ldots, r_{m}$ are allocated to node $R$ and $n$ land use cells $s_{1}, s_{2}, \ldots, s_{n}$ are allocated to node $S$. The travel cost from origin $R$ to destination $S$ along the shortest path for iteration $i$ can be calculated as

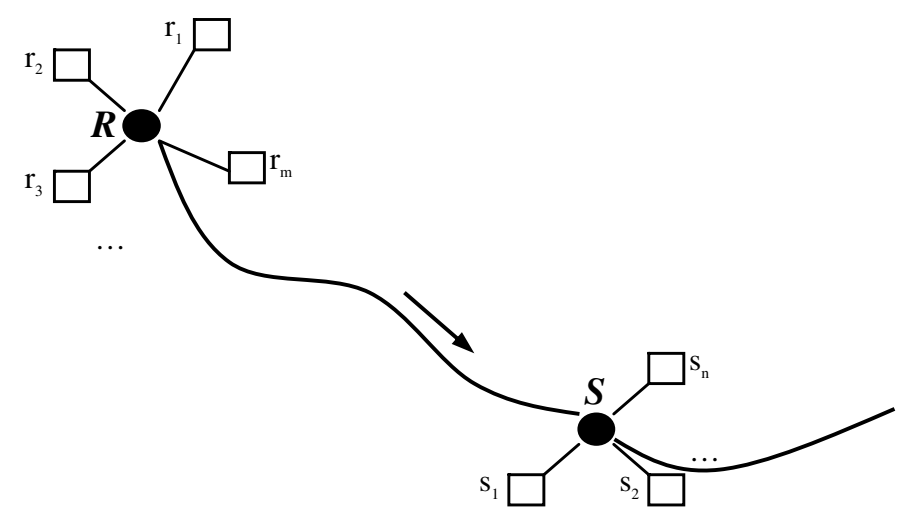

Fig. 2. Calculation of the generalized cost between two nodes. 


$$
c_{R S}^{i}=1 / m \sum_{j=1}^{m}\left(\eta d_{r_{j}} / v_{0}\right)+\sum_{a} c_{a}^{i} \delta_{a, R S}^{i}+1 / n \sum_{k=1}^{n}\left(\eta d_{s_{k}} / v_{0}\right),
$$

where the first part on the right-hand side of the equation calculates the average access cost walking from the land use cells allocated to node $R$ to this node. The variable $d_{r_{j}}$ represents the distance from a land use cell $r_{j}$ to node $R$. The variable $v_{0}$ is a specified minimal speed, which can be interpreted as the speed for accessing the closest network nodes from the land use layer. Similarly, the third part calculates the average access cost from node $S$ to the land use cells attached to it. The second part sums the generalized costs of the links in $\left\{\boldsymbol{P}_{R S}\right\}$ where $\delta_{a, R S}^{i}$ is a dummy variable equal to 1 if a link belongs to $\left\{\boldsymbol{P}_{R S}\right\}$ and 0 otherwise.

Similar to Eq. (2), the travel cost from a land use cell $r_{j}$ attached to origin $R$ to a land use cell $s_{k}$ attached to destination $S$ along the shortest path for iteration $i$ can be calculated as

$$
c_{r_{j} s_{k}}^{i}=\eta d_{r_{j}} / v_{0}+\sum_{a} c_{a}^{i} \delta_{a, R S}^{i}+\eta d_{s_{k}} / v_{0} .
$$

A trip table (origin-destination matrix) is computed using a gravity-based trip distribution model (Hutchinson, 1974). The number of trips from origin $R$ to destination $S$ is calculated as

$$
q_{R S}=g_{R} h_{s} \mathrm{e}^{-\theta c_{R S}} / \sum_{Q \in\{V\}, Q \neq R} h_{Q} \mathrm{e}^{-\theta c_{R Q}},
$$

where $g_{R}$ is trips produced from origin $R$. The variable $h_{S}$ represents trips attracted to destination $S$. The variable $Q$ is any node in the network other than $R$. The variable $\theta$ is a specified friction factor representing the disinclination to travel as travel cost rises.

The investment process incorporates links as agents that make their independent economic decisions according to simple, myopic rules. In the reality, two nodes can be connected either by two neighboring links with opposite directions, say link $a$ from node $R$ to node $S$ and link $b$ from node $S$ to node $R$, or by a one-way link, say link $a$ only. In the latter case, however, there may arise the issue that travelers cannot reach $S$ from $R$ on the network. To ensure the connectivity of a network throughout a decline phase, this study focuses on networks that are connected only by two-way links. This study also assumes that links $a$ and $b$ are operated at the same speed level by a single agent. The reason is twofold. First, in practice, both directions of a segment are usually built simultaneously and maintained at the same service level. Second, if the two parallel and opposite links connecting two nodes operate separately, they may develop into different service levels. It is obviously not reasonable to remove both links from the network at a time (since one-way links are not allowed) just because one of them is weak. Thus the single-agent assumption is made to bond the two links. Let $f_{a}^{i}$ and $f_{b}^{i}$, respectively, represent the flows of link $a$ and link $b$ for iteration $i$. The variable $v_{a b}^{i}$ represents the speed of both links for iteration $i$. An agent operates the two links as a whole, gathering revenues from both links, maintaining them according to their respective costs, and then (dis)investing in the speed of the two links for each iteration, respectively, according to three following component models: a revenue model, a cost model, and an investment model.

\subsection{Revenue model}

Tolls are collected by links according to the length of link, the flow traversing the link and the speed level. The toll revenue gathered by the agent operating link $a$ and link $b$ for iteration $i$ is calculated as follows:

$$
E_{a b}^{i}=\tau\left(l_{a b}\right)^{\rho_{1}}\left[\left(f_{a}^{i}\right)^{\rho_{2}}+\left(f_{b}^{i}\right)^{\rho_{2}}\right]\left(v_{a b}^{i}\right)^{\rho_{3}},
$$

where $l_{a b}$ is the length of both links given that link $a$ and link $b$ have the same length.

\subsection{Cost model}

The cost to maintain a link in its present usable condition depends on its length, flow, and speed. The cost of a link is divided into fixed and variable components. Fixed cost is independent of flow and speed and only 
related to link length. The cost spent by the agent operating links $a$ and $b$ for iteration $i$ is calculated by adding up the fixed and variable costs spent by both links as

$$
C_{a b}^{i}=C_{\text {fixed }, a b}^{i}+C_{\mathrm{var}, a b}^{i}=\left[2 \mu_{2}\left(l_{a b}\right)^{\alpha_{1}}\right]+\left[\mu_{1}\left(l_{a b}\right)^{\alpha_{1}}\left[\left(f_{a}^{i}\right)^{\alpha_{2}}+\left(f_{b}^{i}\right)^{\alpha_{2}}\right]\left(v_{a b}^{i}\right)^{\alpha_{3}}\right],
$$

where $\mu_{1}$ is the variable cost rate and $\mu_{2}$ is the fixed cost rate. The coefficients $\alpha_{1}, \alpha_{2}$, and $\alpha_{3}$ are the length power, flow power, and speed power specified in the cost model.

\subsection{Investment model}

Each link (agent) is assumed to spend all available revenue at the end of each iteration without saving for the next time step. If the revenue gathered from link $a$ and link $b$ for iteration $i$ is greater than the maintenance cost spent, i.e., $E_{a b}^{i}>C_{a b}^{i}$, remaining revenue will be invested to increase its running speed. As a result, the speed of both links increases. In contrast, if the revenue is insufficient to cover the cost, the speed drops. This simple investment policy adopted by each agent in the network can be expressed as

$$
v_{a b}^{i+1}=v_{a b}^{i}\left(E_{a b}^{i}-C_{\mathrm{fixed}, a b}^{i} / C_{\mathrm{var}, a b}^{i}\right)^{\beta},
$$

where $\beta$ is a specified speed improvement coefficient.

\section{Performance measurement}

The performance of a transportation system has been the subject of interest to a wide range of professions, each with unique concerns and objectives (Levinson, 2003). Viewing a transportation network as a single market, economists aim to maximize the total social welfare system wise, while local government and city planners may care about only the surplus of travelers, given that all the links are assumed to be private in this study. In recent years, more and more transportation planners and engineers aim to improve accessibility (Hanson, 1986; Niemeier, 1997), which refers to the ease with which desired destinations may be reached via a network, taking into account both transportation and land use of a region. Employing our simulation model, performance measures are developed from different perspectives and traced throughout the decline phase of a network to examine how decentralized decisions of travelers and autonomous links drive the rise and fall of a network over space and time, as well as how different professions could tackle this process to approach their respective objectives.

\subsection{Consumers' surplus}

On a transportation network, travelers enjoy consumers' surplus when the generalized travel cost (including travel time and toll) that they actually pay for traveling is lower than that they would be willing to pay. Fig. 3 plots the conceptual demand curve and supply curves for a pair of land use cells in a single transportation market. We assume that the number of trips from the origin cell $r$ to the destination cell $s$ decreases with

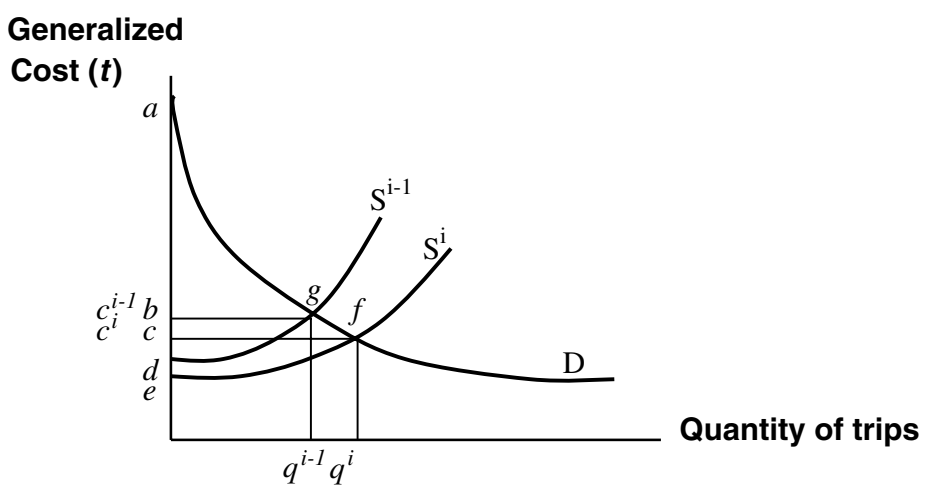

Fig. 3. The demand curve of a pair of land use cells. 
the increase of the generalized travel cost between two cells. This relation defines a downward demand curve $D$ of the O-D pair. Curves $S^{i-1}$ and $S^{i}$ are the supply curves of two consecutive iterations $i-1$ and $i$, respectively. For iteration $i-1, q^{i-1}$ trips are generated from cell $r$ to cell $s$ at the generalized cost $c^{i-1}$ upon the equilibrium between the demand and supply. Consumer surplus enjoyed by travelers from the origin cell $r$ to the destination cell $s$ is represented as area $a-e-b$ for iteration $i-1$ while as area $a-f-c$ for iteration $i$. Assuming the portion of demand curve from $e$ to $f$ is linear, the change of consumer surplus from iteration $i-1$ to $i$, i.e., area $b-e-f-c$, can be approximated using the "rule of $1 / 2$ " (Neuberger, 1971), which indicates that where demand changes in response to the increase or decrease in costs, with relatively small changes in costs, the convention is to attribute half of the change in costs to the trips lost or gained. The total change of consumers' surplus on a network is then calculated by adding up those changes of all cell pairs as follows:

$$
\Delta U^{i}=U^{i}-U^{i-1}=\sum_{r-s}\left[0.5\left(c_{r-s}^{i-1}-c_{r-s}^{i}\right)\left(q_{r-s}^{i}+q_{r-s}^{i-1}\right)\right]
$$

where $r-s$ is a pair of land use cells on the land use layer.

Note that the linear assumption for the rule of $1 / 2$ only stands over small changes on the demand curve and may be violated by changes in the structure of the network. This explains why the land use cell instead of link or node is selected as the geographical unit upon which we approximate the changes of consumers' surplus by pairs. Different from permanent land use cells in our model, both links and nodes are removable during the degeneration process. When a link or a node is removed, all the trips currently associated to the link or node will immediately be re-allocated to remaining links or node pairs, resulting in an abrupt rightward shift of the demand curves of links or node pairs. In this case, the small change assumption no longer stands and we cannot approximate the change of consumers' surplus using the "rule of $1 / 2$ ".

\subsection{Welfare}

Total social welfare is defined here as consumers' surplus plus profit. Autonomous links supply services (road bed, pavement, track, speed, etc.) at different levels and earn their profit when the revenue (toll) they collect from travelers are in excess of the cost of providing these services. The earning of each link agent can be calculated by subtracting its maintenance cost from its revenue according to Eqs. (5) and (6), then the earnings of all existing agents are added up to the total profit $\Pi$. The change of total profit from iteration $i-1$ to iteration $i$ can be calculated as

$$
\Delta \Pi^{i}=\Pi^{i}-\Pi^{i-1}=\sum_{a b}\left[\left(E_{a b}^{i}-C_{a b}^{i}\right)-\left(E_{a b}^{i-1}-C_{a b}^{i-1}\right)\right],
$$

where $a b$ is any existing link-agent on the network with two opposite links $a$ and $b$.

The change of total social welfare from iteration $i-1$ to $i$ is then calculated as

$$
\Delta W^{i}=\Delta U^{i}+\Delta \Pi^{i} .
$$

We also calculate the cumulative change of total social welfare from a specific starting point $i^{*}$ as

$$
\Delta W^{i}=\sum_{k=i *}^{i}\left(\Delta U^{k}+\Delta \Pi^{k}\right) .
$$

\subsection{Accessibility}

A variety of accessibility measures have been developed in different fields, among which the gravity-type measure (Basmaciyan and Schmidt, 1964; Handy, 1992) is perhaps most widely adopted. This type of measure is typically expressed as a function of available opportunities moderated by some measure of impedance which takes the following form:

$$
A=\sum_{R} \sum_{S} f\left(K_{S}, c_{R S}\right)
$$


where $K_{S}$ is a measure of the attractiveness of the destination node $S$, while $c_{R S}$ the generalized cost of travel from $R$ to $S$, referring to Eq. (2). A typical analytical expression of the function can be either in an exponential form or a Newtonian quadratic form of the generalized cost. This study chooses the latter for simplicity:

$$
A_{R}=\sum_{S} h_{S}\left(c_{R S}\right)^{-2}
$$

where the number of trips attracted to node $S\left(h_{S}\right)$ is used to indicate the attractiveness of this node, referring to Eq. (4).

\section{Experiments}

A series of five experiments were carried out on a hypothetical surface transportation network with the same initial conditions, including the same set of link speeds randomly distributed between 1 and 10, and the same set of uniform land uses (resulting in 10 trips generated from and 10 trips attracted to each cell per day). The hypothetical network is called the $4 \times 4$ complete network, which is developed by directly connecting every pair of two nodes among an original node set $\left\{V^{*}\right\}$ that come from the intersection nodes of a $4 \times 4$ grid network. When links intersect, a new secondary node is created, and the longer link is replaced by shorter links that ultimately connect the same original nodes. A secondary node is not necessarily directly connected to an original node or another secondary node. Where links overlap, the longer link is eliminated. A complete network represents a highly redundant network that is expected to decline over time.

All the experiments conducted in this study shared the following set of degeneration criteria to delete the weakest $\operatorname{link}(\mathrm{s})$ from the network at each time period.

First, the weakest link among existing removable links is killed at the beginning of iteration $i$. A removable link is one which, by its removal, does not disconnect the network. Different rules to select the weakest link will be discussed later in this section.

Second, one two-way link is killed at a time. Once a link from node $R$ to node $S$ is killed, the other (parallel and reverse) link from node $S$ to node $R$ operated by the same agent will be automatically killed. Thus two links will be killed at a time. This rule ensures a minimal number of links to be killed in each round.

Third, if a node exists, it must be connected to at least one existing node. Isolated nodes and sub-networks are not allowed for the whole network.

Experiments are summarized in Table 1, and Table 2 lists model parameters and their values specified for the experiments. As can be seen, the experiments only differ on when to start and when to stop executing the degeneration process, as well as how to select the weakest link(s).

As shown in Table 1, Experiments A1-A3 differ only on when a degeneration process is implemented. Before the degeneration process is started, a network grows with its structure fixed, similar in nature to those presented in Yerra and Levinson (2005) and Levinson and Yerra (2006), and the equilibrium of growth is reached when the network does not change in two consecutive iterations. Since all the experiments reached the equilibrium of network growth within 20 iterations, the 20th iteration was specified as the starting point of the degeneration process, if any, for all the experiments. Experiment A1 never started a degeneration process. Thus the network stayed stable after the network growth reached equilibrium. Experiment A2 started the

Table 1

Specifications of experiments

\begin{tabular}{llll}
\hline No. & Degeneration process & & Iterations \\
\cline { 2 - 4 } & Start & End & Indicator \\
\hline A1 & Never & NA & Speed \\
A2 & After network growth reaches equilibrium & When minimally connected network is obtained & 850 \\
A3 & & Right after the cumulative change of total welfare peaks & Speed \\
B & & When minimally connected network is obtained & Volume \\
C & & & B/C ratio \\
\hline
\end{tabular}


Table 2

Model parameters and their specified values

\begin{tabular}{lll}
\hline Parameters & Description & Value \\
\hline$\rho_{1}$ & Length coefficient in Eqs. (1) and (5) & 1.0 \\
$\rho_{2}$ & Flow coefficient in Eqs. (1) and (5) & 1.0 \\
$\rho_{3}$ & Speed coefficient in Eqs. (1) and (5) & 0.0 \\
$\tau$ & Toll rate in Eqs. (1) and (5) & 1.0 \\
$\eta$ & Value of time in Eqs. (1)-(3) & 0.6 \\
$v_{0}$ & Walking speed in Eqs. (2) and (3) & 0.01 \\
$\theta$ & Friction factor in Eq. (4) & 0.01 \\
$\mu_{1}$ & Variable cost rate in Eq. (6) & 1.0 \\
$\mu_{2}$ & Fixed cost rate in Eq. (6) & 2.0 \\
$\alpha_{1}$ & Length power in Eq. (6) & 1.0 \\
$\alpha_{2}$ & Flow power in Eq. (6) & 0.75 \\
$\alpha_{3}$ & Speed power in Eq. (6) & 0.75 \\
$\beta$ & Speed improvement coefficient in Eq. (7) & 1.0 \\
\hline
\end{tabular}

degeneration process at the 20th iteration, and terminated it when the minimally connected network is derived. Experiment A3 stopped the degeneration when the cumulative change of total welfare peaks in A1.

The experiments A2, B, and C differ only on the indicator used to evaluate the performance of individual links and identify the weakest. Three plausible indicators are tested. Note that the degeneration model assumes a link and its neighboring (parallel and reverse) link are operated by a single agent at the same speed. Experiment A2 kills the link with the lowest speed among all the existing removable links and automatically kills its neighboring link. Experiment B kills the two links operated by the agent with the lowest through-traffic volume (the sum of the traffic on both links). Experiment $C$ kills the agent operated at the lowest benefit-cost ratio (BCR). Referring to Eqs. (5) and (6), the BCR for the agent operating links $a$ and $b$ at iteration $i$ is calculated as

$$
\mathrm{BCR}=E_{a b}^{i}-C_{\text {fixed }, a b}^{i} / C_{\mathrm{var}, a b}^{i},
$$

where $E_{a b}$ is the revenue collected from links $a$ and $b, C_{\text {fixed, } a b}$ is the fixed cost spent on $a$ and $b$, and $C_{\mathrm{var}, a b}$ is the variable cost.

\section{Results}

Fig. 4 illustrates the fluctuations of the cumulative change of total social welfare derived from Experiments A1-A3. As we begin to calculate the cumulative change of welfare at the 20th iteration when the degeneration process, if any, is started, $i^{*}$ is equal to 20 in Eq. (11). As a plausible approximation, the change of welfare is calculated every five iterations to save running time. In other words, each curve demonstrates the cumulative change of welfare from the end of the 15th iteration every five iterations. Note also that three curves start from the same point with the same cumulative change at the 20th iteration because the three experiments went through exactly the same network growth process before the degeneration was started at this iteration.

Experiment A1 specifies the network growth model without degeneration. The A1 curve starts at the 20th iteration where the cumulative change of welfare equals -576.1 , indicating the welfare decreases about 576 units since the 15th iteration. Since the network reached equilibrium before the 20th iteration, the A1 curve remains flat in the following iterations. The fact that the welfare declines as the network evolves between the base year and the equilibrium year (given that cumulative welfare change remains negative) suggests that most links in this network cannot maintain their specified initial service levels, and that removing the inefficient links from the network could improve the overall efficiency.

Experiment A2 starts degeneration at the 20th iteration and executes the degeneration process until completion. The cumulative welfare unsurprisingly keeps increasing and reaches the maximum at the 65th iteration. Since the degeneration process is enforced in this experiment to remove links each round regardless of the network efficiency, some useful links have to be removed from the remaining network after this turning point, pulling the welfare down dramatically. After the degeneration process is stopped at the 805th iteration when 


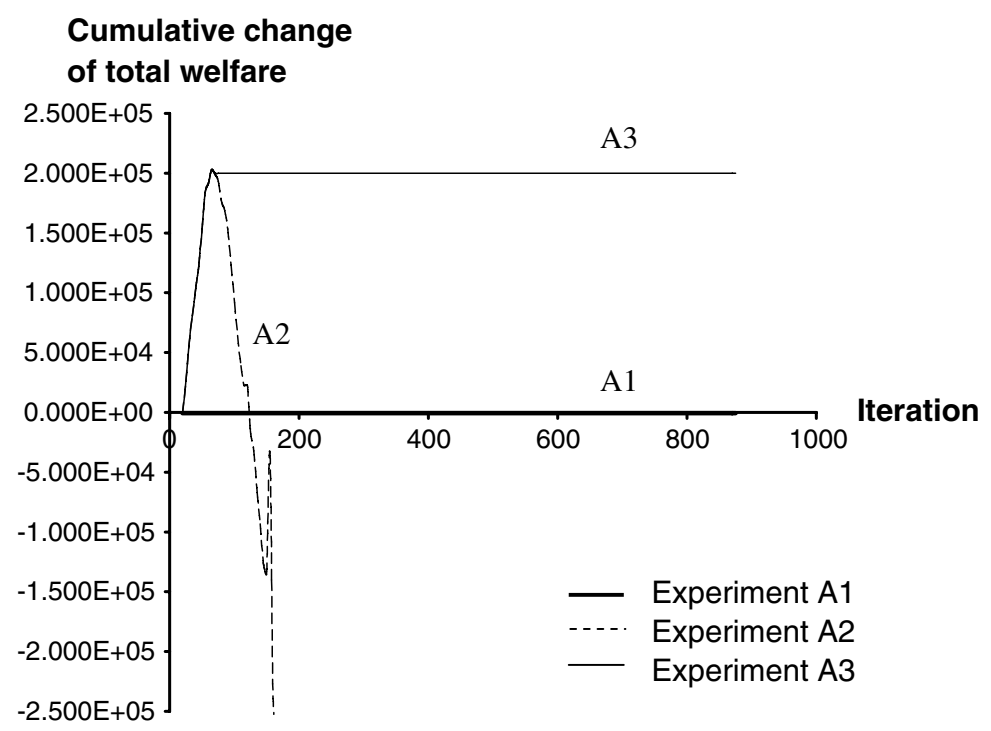

Fig. 4. The cumulative change of welfare (Experiments A1-A3).

the minimally connected network remains, the network becomes unchanged again until the end of the experiment. The final cumulative change of total welfare $\left(-1.497 \times 10^{9}\right.$ uunits $)$ is much lower than the peak value at the 65 th iteration $\left(2.029 \times 10^{5}\right.$ units $)$.

Experiment A3 also starts degeneration at the 20th iteration but stops it as long as the welfare peaks at the 65th iteration. Before this point the A3 curve overlaps with the A2 one. After the degeneration process is terminated, the network soon finds a new stable status after several iterations. During this short period individual links quickly adjust their service levels, and travelers quickly adjust their route choices as well. The stable welfare level $\left(2.000 \times 10^{5}\right.$ units $)$ is slightly lower than the maximal welfare it has achieved at the 65th iteration. Since then the network is fixed, which we called the optimal network topology in terms of total social welfare under the specific degeneration criteria.

The results of Experiments A1-A3 together reveal how the degeneration process could change the economic efficiency of a redundant network. The results of Experiment A2 indicate that the degeneration process can improve the total social welfare by removing inefficient links, but if a network becomes compact enough, further degeneration will impair the welfare, which we would call "over-degeneration". Experiment A3 suggests a way for economists to avoid over-degeneration and derive a relatively efficient transportation system, even though the market is completely autonomous and decentralized, by tracking down the decline phase of the system and terminating the degeneration process at the point when the cumulative change of total social welfare peaks.

To illustrate the decline process of the complete network simulated in Experiments A1-A3, Fig. 5 displays the snapshots of the network at different stages: the initial network (iteration 0), the network at equilibrium before degeneration (iteration 20), the "optimal" network topology with regard to the total social welfare under specified degeneration criteria (iteration 65), and the minimally connected network at the end of the degeneration process (iteration 850). Different speed levels of links are displayed as different boldness and grayness.

While economists want to maximize the total social welfare of a network, policy makers, planners, and engineers may have different objectives, for instance, to maximize consumers' surplus or accessibility (given the network infrastructure is completely privatized in our case). Fig. 6 illustrates the fluctuations of accessibility for Experiments A1-A3. The accessibility of the network remains at 15,003 units in Experiment A1 after the first 20 iterations. As the degeneration process is executed throughout Experiment A2, the accessibility consistently drops to 4098 units at the end of the experiment. No increase is observed as in the fluctuation of total social welfare. Experiment A3 stops the degeneration process at the 65th iteration when the maximal welfare is reached, and the network is quickly stabilized on a slightly lower level of accessibility. The decrease is so small (0.03) that it is hardly noticeable in Fig. 6. These results, compared to those in Fig. 4, suggest that 


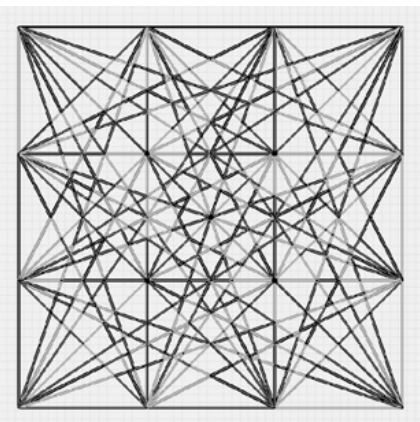

(1)

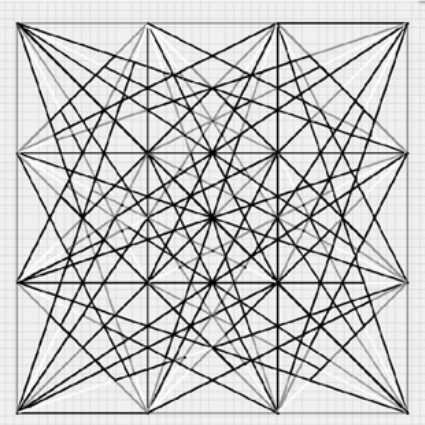

(3)

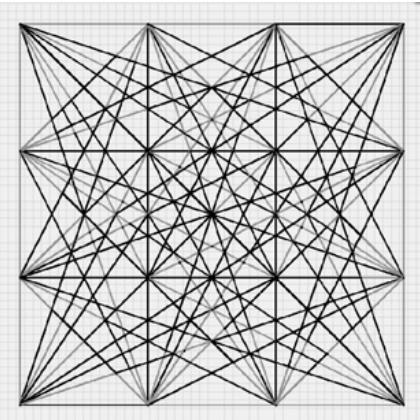

(2)

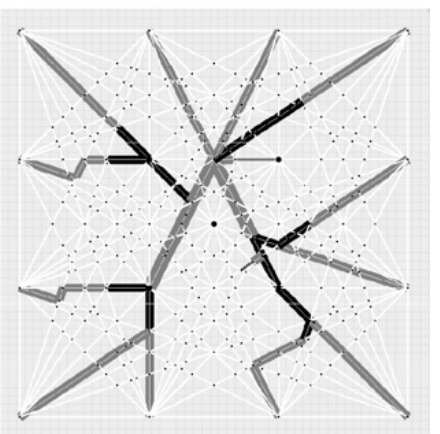

(4)

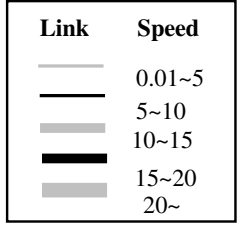

Fig. 5. Snapshots of the declining network. (1) The initial network (iteration 0) (2) The network topology at equilibrium before degeneration (iteration 20) (3) The optimal network topology (iteration 65) (4) The minimally connected network (iteration 850).

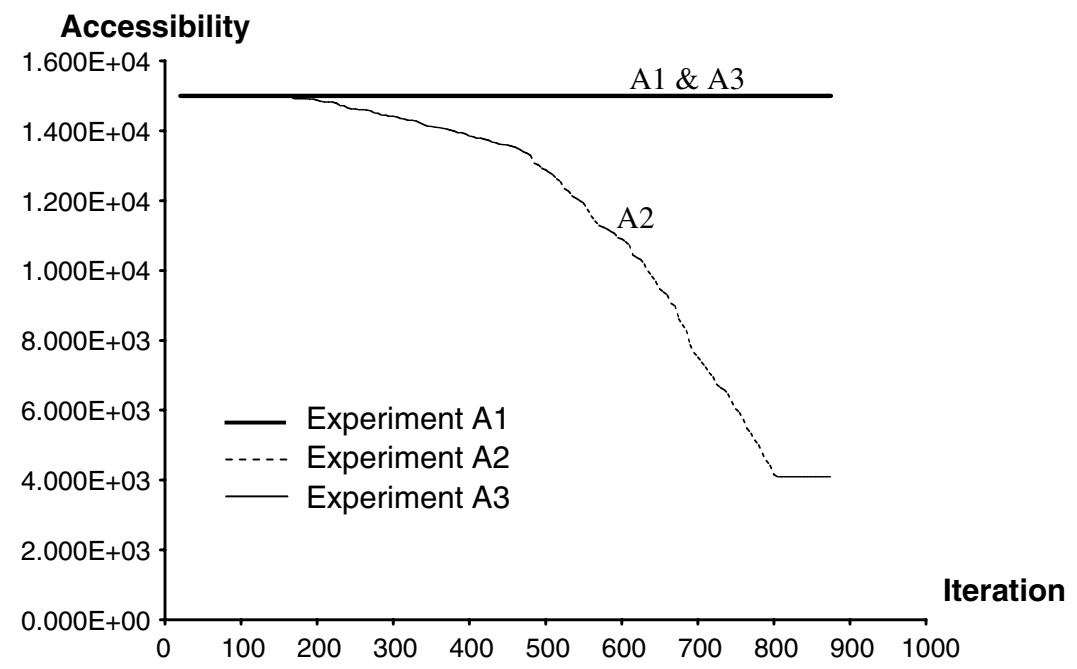

Fig. 6. The change of accessibility (Experiments A1-A3).

the degeneration process affects the accessibility and total social welfare of a network differently: it always impairs the overall accessibility because the removal of links and nodes from the network unavoidably reduces travelers' flexibility in choosing their routes and destinations and accordingly increases their cost spent on travel, while it may improve the total welfare of the network by removing profitless links at the beginning, because the savings on the deficit (at least in a myopic term) of these removed links may compensate the loss of travelers, and thus benefits the system as a whole. Similar to the measure of accessibility, consumers' surplus is also consistently dropping as the degeneration process is executed in Experiments A2 and A3, suggesting that this process always impairs travelers' benefits. 


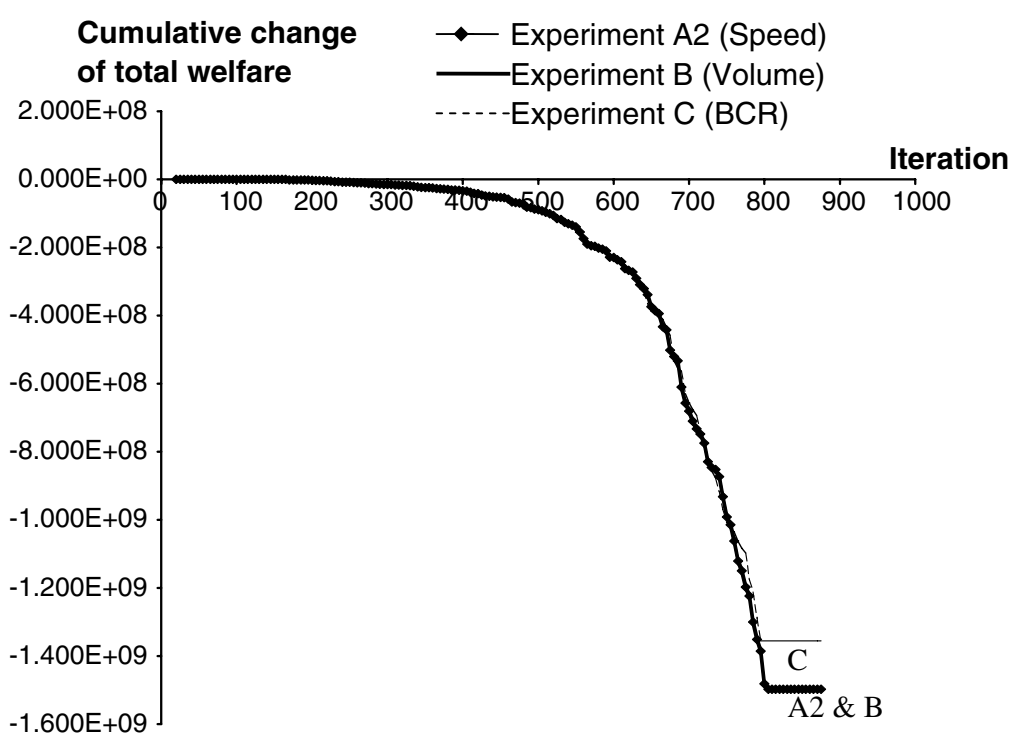

Fig. 7. The cumulative change of welfare (Experiments A2, B, and C).

The results of Experiments A2, B, and C, shown in Fig. 7, exhibit similar fluctuations of welfare over time (especially at the beginning of the degeneration process), suggesting that the evolution of network efficiency is not sensitive to different evaluation criteria that determine the weakest link(s) during the degeneration process of a network.

\section{Conclusions}

The contribution of the paper to the literature is that for the first time it carried out a theoretic exploration into the economic mechanisms behind the decline of a surface transportation network at a microscopic level, and modeled this process based on the assumption that the decline phase is a spontaneous process driven by completely decentralized and autonomous decisions of individual travelers and privatized infrastructure segments (links). The decline of a network is enabled by the degeneration process by which the weakest link(s) is removed iteratively from the network according to simple evaluation criteria of link performance.

Our simulation model can be also thought of as a monopolistically competitive model, which corroborates the phenomenon of spatial competition over an urban space (Christaller, 1933). Viewing a transportation network as a single market with the total travel demand fixed, as profitless links are shut down, the market demand of the remaining links increases, such that efficient links will be further reinforced while less efficient links will be degenerated until abandoned. Likewise, as nodes that connect to failed links are shut down, the market area of land uses they originally occupy will be taken by neighboring surviving nodes, which then become more attractive to travelers. Consequently, hierarchical links and places emerge over time. During this process, the rise and fall of link rents (profitability) triggers the removal of failed links and nodes, and the change of market density in turn amplifies the differentiation of link rents. A model of co-evolution of a transportation network and land use could incorporate land use dynamics in response to the evolution of the network, with the potential to better interpret the spatial competition over network and urban space (Levinson et al., 2006). Moreover, inspired by the spatial patterns of hierarchical places that have been suggested in central place theory (Christaller, 1933; King, 1985), we have employed our degeneration model to examine the spatial patterns of hierarchical transportation networks that emerge over time and space (Xie and Levinson, 2005).

Experimental results show that a turning point exists during the degeneration process regarding total social welfare: when inefficient links are removed from a redundant network, the total welfare is improved, while if the degeneration process persists at a point so that profitable links have to be removed, the economic efficiency of the network will be impaired. The experiments show that if the degeneration process is stopped at the 
turning point, a relatively efficient network topology can be derived in terms of maximizing the total social welfare during the decline phase. On the other hand, travelers are always worse off during the decline phase, from the perspective of either consumers' surplus or network accessibility, as the removal of links and nodes reduces their flexibility and increases their cost of travel. These findings can be of interest to economists and policy makers who have to tackle the shrinking infrastructure of a transportation mode while maintaining the performance of the system.

Aside from directly describing the abandonment of links in an over-invested transportation network, degeneration can have several other interpretations in transportation studies. It can represent an underdeveloped area where all point-to-point paths can be used, and some which are more valuable are made faster while others are abandoned over time. It can also be used as a model wherein a set of many potential network additions are considered and degeneration is used to winnow that set. In these cases, the degeneration process is not limited to the decline phase of the life cycle that it literally resembles. Instead, it is coupled with a growth process in which a system grows and declines with important components reinforced while weak components deprecated.

The simplified demand model adopted in this study assumes sufficient capacities on links and thus ignores the congestion effect. This simplification is plausible for a theoretical exploration into the long-run evolution of an over-invested network, and largely reduces the complexity of our simulation model. But a more sophisticated travel demand model that considers congestion delay, system equilibrium, and perhaps perceived travel time on a congested network, such as Deterministic User Equilibrium model and Stochastic User Equilibrium model (Sheffi, 1985), will improve certain aspects of the realism in the model, at the cost of increased complexity.

\section{Acknowledgements}

The authors acknowledge Bhanu M. Yerra, whose research work provides a substantial foundation to this study. This material is based upon work supported by the National Science Foundation under Grant No. 0236396. All opinions and errors remain those of the authors.

\section{References}

Barabasi, A., Bonabeau, E., 2003. Scale-free networks. Scientific American 288 (5).

Barabasi, A., Albert, R., Jeong, H., 1999. Scale-free characteristics of random networks: the topology of the world wide web. Physica A 272, 173-187.

Basmaciyan, H., Schmidt, J., 1964. Development and application of a Modal split model for the Puget Sound Region, PSRTS Staff Report, 12, Seattle, Washington.

Boyce, D.E., Farhi, R., Weischedel, R., 1974. Optimal Subset Selection: Multiple Regression, Interdependence and Optimal Network Algorithms. Springer, New York.

Chachra, V., Ghare, P.M., Moore, J.M., 1979. Applications of Graph Theory Algorithms. North-Holland, New York.

Christaller, W., 1933. Die zentralen Orte in Süddeutschland. Gustav Fischer, Jena. Translated by Charlisle W. Baskin as Central Places in Southern Germany. Prentice Hall, Englewood Cliffs, NJ, 1966.

Fullerton, B., 1975. The Development of British Transport Networks. Oxford University Press, London.

Garrison, W., Levinson, D., 2006. The Transportation Experience: Policy, The Transportation Experience: Policy. Oxford University Press, New York.

Handy, S., 1992. Regional versus Local Accessibility: Variations in Suburban Form and the Effects on Non-work Travel. Ph.D. dissertation, University of California, Berkeley, CA.

Hanson, S., 1986. The Geography of Urban Transportation. The Guilford Press, New York.

Helbing, D., Keltsch, J., Molnár, P., 1997. Modeling the evolution of human trail systems. Nature 388, 47.

Hilton, G., Due, J., 1960. The Electric Interurban Railways in America. Stanford University Press, Stanford.

Hutchinson, B.G., 1974. Principles of Urban Transportation Systems Planning. McGraw-Hill, New York.

King, L., 1985. Central Place Theory. Sage, London, pp. 28-46.

Krugman, P., 1996. The Self-Organizing Economy. Blackwell, Malden, pp. 53-100.

Levinson, D., 2003. Perspectives on efficiency in transportation. International Journal of Transport Management 1, 145-155.

Levinson, D., Yerra, B., 2006. Self organization of surface transportation networks. Transportation Science 40 (2), $179-188$.

Levinson, D., Xie, F., Zhu, S., 2006. The co-evolution of land use and transportation. ISTTT 17-253 Paper to be presented at the 17th International Symposium on Transportation and Traffic Theory (ISTTT), London, July 23-25, 2007.

Mensch, G., 1979. Stalemate in Technology: Innovations Overcome the Depression. Ballinger, New York. 
Nakicenovic, N., 1988. Dynamics and replacement of U.S. transport infrastructures. In: Ausubel, J.H., Herman, R. (Eds.), Cities and their Vital Systems: Infrastructure-Past, Present and Future. National Academy Press, Washington, DC.

Neuberger, H., 1971. User benefit in the evaluation of transport and land use plans. Journal of Transportation Economics and Policy, 52-77.

Newman, M., 2003. The structure and function of complex networks. SIAM Review 45, 167-256.

Niemeier, D., 1997. Accessibility: an evaluation using consumer welfare. Transportation 24, 377-396.

Ortuzar, Juan de Dios, Willumsen, L.G., 2001. Modeling Transport. Wiley, West Sussex.

Schelling, T.C., 1978. Micromotives and Macrobehavior. W.W. Norton, New York.

Schweitzer, F., Ebeling, F., Rose, H., Weiss, O., 1998. Optimization of road networks using evolutionary strategies. Evolutionary Computation, 419-438.

Sheffi, Y., 1985. Urban Transportation Networks: Equilibrium Analysis with Mathematical Programming Methods. MIT Press, Cambridge, pp. 287-289, 325-332.

Vaughan, R., 1987. Urban Spatial Traffic Patterns. Pion, London, pp. 96-155.

Xie, F., Levinson, D., 2005. The topological evolution of road networks. 07-0671 Paper to be presented at the 86th Annual Meeting of the Transportation Research Board, Washington, DC, January 21-25, 2007.

Yamins, D., Rasmussen, S., Fogel, D., 2003. Growing urban roads. Networks and Spatial Economics 3, $69-85$.

Yang, H., Bell, M.G.H., 1998. Models and algorithms for road network design: a review and some new developments. Transport Reviews $18,257-278$.

Yerra, B., Levinson, D., 2005. The emergence of hierarchy in transportation networks. Annals of Regional Science 39 (3), $541-553$.

Zhang, L., Levinson, D., 2004. A model of the rise and fall of roads. Presented at MIT Engineering Systems Symposium. 